\title{
'Mathematical mosaic'
}

\section{Cecil Balmond}

The Spiral challenges the concept of a museum: does space have to be container-like and neutered to house work of art? When there is much invention and fantasy in porcelain or jewellery or the lines of a fashion garment, should the space around the exhibits be inanimate; is not the real invention to present art, not as lost object in a static box container, but as vital trigger in a spatial dynamic?

With a shape that is formless and a façade that motivates geometry as a mathematical mosaic, the V\&A Spiral designed by Daniel Libeskind opened the debate: Norm and Form would have new definitions from the year 2000 onwards (Fig 1).

A cherished symmetry and insistence of right-angled forms rejected, and the old paradigm of fixed centre left behind, the V\&A Spiral vaults into new space. Inside is outside. Floors are denied columns, and walls offer no vertical short cuts for gravity. Structure and architecture become one immediacy.

\section{The Beginning}

I looked into the form without really knowing it at first; I saw walls flying across space. The tilting planes climbed and cut into each other, violent, shattering any notion of building in the conventional sense. And the dialogue began between Daniel Libeskind and myself, how could such a form be built?

There were two ways to consider the question: implant a certain massiveness and cele- brate a high redundancy in the configuration; or trap the tilting planes in a modern rationale of discrete 'framing'.

The former would give concrete as a material of tradition, used in an extreme definition; the latter would reduce the great planes to a framing buttressed by internal stiffeners and cross bracing. One method provides density, opacity and three-dimensional surface as structure, the other lightness and openness that is then clad and windowed. The first answer leads to a labyrinth, the second to transparency.

If the form were closed, it could be a mineral deposit, or, if an open transparent steel framed building, it could be a lantern or a bea-
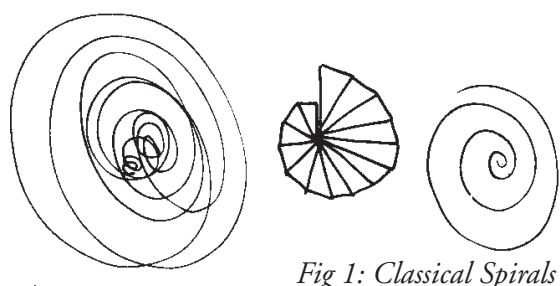

Fig 1: Classical Spirals 
con. If it were heavy, could it be hacked out of granite, or was it buildable out of special masonry? The images helped loosen the thinking and inspired us to look for the radical.

There was no science to it, the instinctive decision was to go for the inscrutable, the closed-in secret. As a check, I mocked up the stick model, trying to find common lines for the vertical structure, attempting to brace diagonally in between edges, but the structure looked forced and contrived. The seamless flow of planes felt inherently better. So we decided on concrete, the artesian material of sand, water and gravel, to 'pour' the form.

\section{Development}

In the first model standing one metre high and built out of stiff white card, the walls leapt dramatically in a stacking of twenty-metresdeep planes, exciting and extravagant. In cutaway mode though, viewed from the inside, something else happened. At five-metre storey heights, floors interrupted the raking planes. The internal spaces became serene, un- cluttered, and with no columns. Light streamed down through the overlaps on plan. A slow shifting of space occurred, around a gradual displacing sense of the vertical.

$$
\begin{aligned}
& \text { outside dramatic and violent } \\
& \text { inside quiet and contemplative } \\
& \text { outside theatrical and extrovert } \\
& \text { inside reflective and introvert }
\end{aligned}
$$

Closing like a secret and opening like a drama - the Spiral caught both progressions. The form was at the singular point of a cusp.

\section{METAPHOR}

For time immemorial a winding form has caught peoples' imagination, its symbol serves as talisman and votive force, the contrary movements seeding a deep mystery and ambiguity. To adepts and magicians spirals were Guardians of the Gate and Protectors of the Centre, to ancient geometers the spiral was a 'Mirabilis'. Through tornadoes and whirlpools, in shells and in the horns of rams, in the petals of flowers, spirals frequent nature;

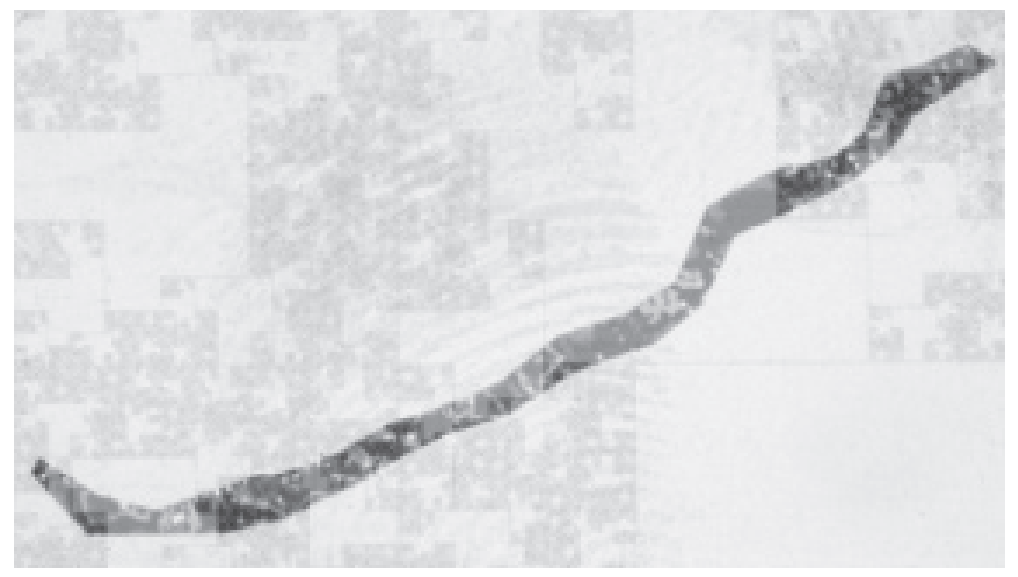

Fig 2: Tiling the spiral... 
spirals turn galaxies and braid DNA strands (Fig 2).

Libeskind and I talked of numbers and their power: of the Eastern legacy of number as abstract and touched with hidden algorithm; and, in the Hebraic tradition, text as number. We talked of serialisation, of music - of the Eastern inspiration for melodic lines that fold and overlay each other, of the Western tradition for harmony and modulation. How these discussions influenced what happened is impossible to tell, but it set off an enquiry with buried codes that helped forge a close partnership.

As Libeskind developed the semantics of spiral I looked at the syntax of connectivity and implied movement. It was not just architecture and engineering but a wider endeavour being molded.

\section{Statics}

The first thoughts I had of structure were in terms of one plane bearing on another, a piggy-back of shared loads working their way down to the ground. Each discrete wall was treated as a mini two-dimensional problem, supported on at least two points. But the model encouraged certain walls to have only one bearing point, and crank in space without support.

Rather than stacked planes, so zigzags came to mind. It felt as if the form wanted to liftoff. The continuity began to grow.

On elevation the model suggested a stacking of tilting planes assembled like a pack of cards, but when looking down the vertical from the top of the form, a linked rotation took shape. A spiral of sorts took hold; something like the staircase in Hitchcock's film 'Vertigo' - a crooked and distorted trajectory.
INTERPRETATION

The oblique planes seemed not to be separate but capable of one gigantic turning, if it was a wrapping, then all the parts could belong to one and the same impulse. And the observation levels at the top, first thought of as a glass box and 'clamped' onto the form, could now logically be the end folds of the trajectory. The conclusion was that the whole twenty three steeply angled planes were but one strip! Folding out the wall planes resulted in a snake-like figure. If the floors attached to the walls were drawn onto the strip, a striped and segmented snake took hold. Each stripe was the length of a floor's connection to the walls that intersected it.

If cuts are made in the strip, both top and bottom, and numbered in sequence, then matching bottom numbers with top ones yields an interlocking form. Like a child's toy the V\&A entrance building is assembled by a set of numbers. In terms of scale, the length of the snake would be the length of Exhibition Road that borders the site, a full 500 metres.

What was the real geometry of such an unravelling? What were the turning patterns of this coiling? We had built the analogue in card and timber, but was there a mathemati$\mathrm{cal}$ algorithm? I was intrigued as to whether a set of simple rules could approach the complexity of the form Was it after all a geometric strategy, albeit of a special sort? With Francis Archer of Arups, I began the search for a simple set of rules that could lead to an interlocking form, arising our of cross-overs on plan - a spiral of sorts. 


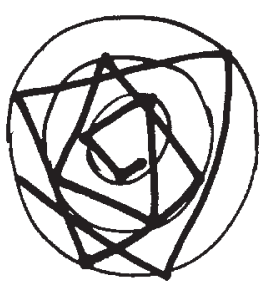

\section{Geometry}

Classical spirals have two models: the logarithmic and the Archimedean. The logarithmic is exponential and is the shape nature favours to mark growth. The Archimedean spiral, named after the famous Greek mathematician, is a constant turning, where the distance from the centre grows in equal jumps like in the windings of a spring.

Both spirals have one thing in common a fixed centre. Classical thinking was based on such stabilisations, the logic of the rule flowing from a fixed and unmoving reference, everything being causal and linear, one spacing relating to the other. A new spiral would be different - non-linear and unpredictable.

\section{Rules FOR A NEW TYPE OF SPIRAL}

- What is needed is a pattern of criss-cross on plan.

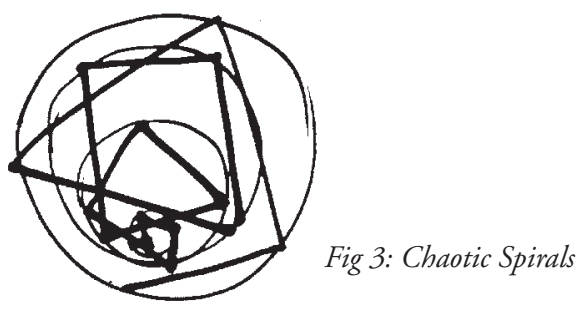

- There can be no fixed centre.

- The centre should be local to a particular level, not a global reference.

- There has to be an erratic tendency which makes the distance of the orbits from the local centre vary.

- The result is to be an unpredictable or chaotic spiral - its trace suddenly jumping and changing radius realising a locus of cross-over.

\section{THE EXPERIMENT}

The answer lies in a drawing that looks like a target. A radius travels around the circles, changing in length and jumping, stopping at points, its origin moving. Connecting the points produces interlock and a sideways drift (Fig 3).

The radius starts to swing around a circle stopping at one point; then moves and turns at similar angles about the centre to other points. At a certain moment the radius shortens and swings around a similar angle as be-
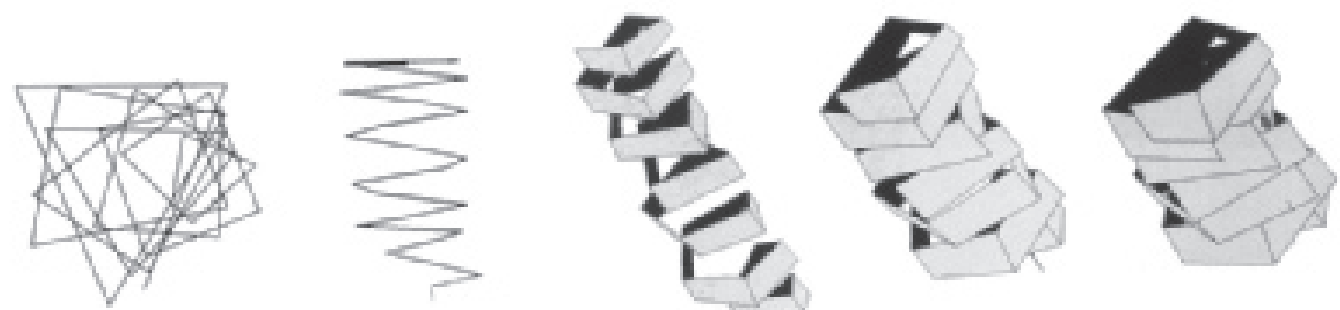

Fig 4: Entering the third dimension the lines become walls 
fore. If this continues with a fixed centre we get a crooked spiral, an imprint of the distorting perspective of the 'Vertigo' stairway. But if the centre moves, as the radius revolves changing its length, the chaotic spiral results. We have an interlocking trace.

To enter the third dimension, the trace is lifted off the ground in a zigzag, each leg rising successively a certain height. The lines become the centre lines of the walls in elevation. Each centre line is now rotated and a normal (a line to 90 degrees to the rotation) projected into space. At right angles to this normal, an infinite plane is swept through the line. The intersection of these planes generate corner fold lines which have mathematical equations. Hence the edge of a wall and its height can be set along these lines of intersection.

By giving a vertical offset distance for both top and bottom corner from the centre line, at each end of a wall, a panel can be formed (Fig 4).

Insisting on intersection is vital for it gives gearing and structure. A serial logic grows. The form transfers loads through its skin needing no other support. The steps of this calculation may be carried out on a spreadsheet.

Because the program is generic, depending on the start point, no two traces would be the same.

If the angle at which the radius spins around is small, then the form evolves smoothly. When the angle at which the radius swings around is large, the trace is spiky, sharp, the consequent form, a densely intersecting shape like a crystalline clump of mineral spars.

What we have is a range of interlocking shapes, all of them built out of rotations and widenings or shortenings, but all arising from a logic of the locus of a point orbiting around a centre, in stepped jumps. In other words, a

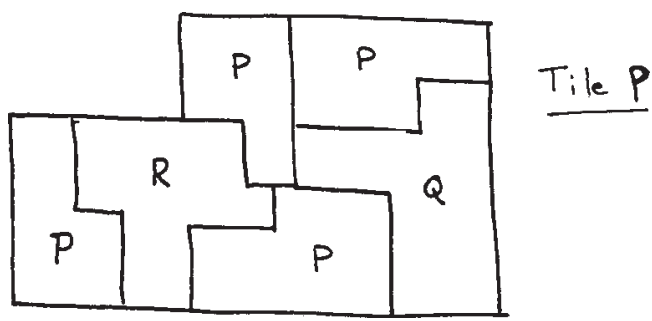

Fig 5: Each 'P' tile contains a mix of $P, Q$ and $R$

spiral, but a generic one, capable of many interpretations.

If buildings today on the High Street are proposed by generating envelopes, a strip that folds at right angles at regular intervals will produce shoe-box like enclosures. The trace is of a repeating closed loop on the horizontal plane. In this scenario a roof is 'cover', a lid.

The V\&A Spiral is also a strip but one that climbs as it folds with no roof. It is finite but unbounded.

\section{Fractile patTERNS}

Victorian patterns, William Morris, the intricacies of the Alhambra and other past poetics came to mind, but a different instinct took hold for the Spiral. We felt the great planes had a movement in space that needed not just 'cover' but augmentation, a vibration of sorts across the walls. It could not be traditional tiling, it had to be something else. I saw a 'shiver' running up the form.

External tiling normally completes the object, refines it and gives the building an ultimate blessing, yet covering the Spiral pointed to another strategy, a kind of mobility that would never complete the building but keep it unfinished always evolving. 


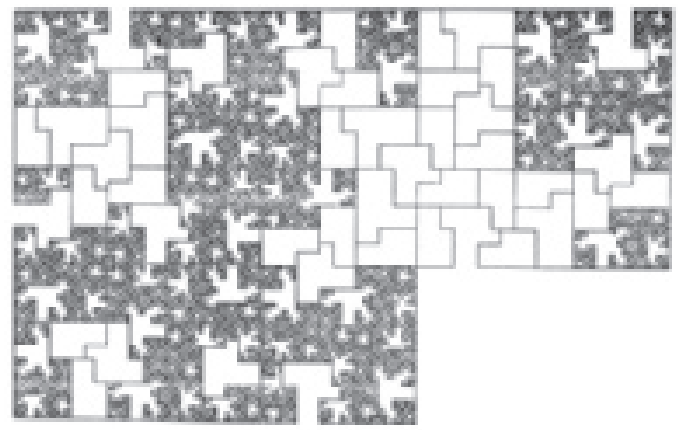

Fig 6: Each Shape contains all three shapes and its pattern repeats infinitely

The idea starts with finding a unit of pattern and using it over and over again in different interlocking adjacencies. What appears to be random will grow to cover the plane. If we know the unit and understand all its flipovers and rotations, as a 'fit' is generated, the notion of 'random' disappears. Serialisation takes over. Instead of static repeating motifs we have movement, a 'charged-up' liveliness. Sequence becomes important. Governed by certain rules of organisation tiling turns into the dynamic of tessellation (Fig 5).

To keep the feeling of sameness and have the potential to be different, I wanted to seed a self-similarity of pattern, necessary to realise these ideas, replicating 'network', and not fixed pattern.

The first shapes that came to mind were distorted pentagons, setting up craggy and spiked patterns, but they seemed too violent and 'one dimensional', not quite in resonance with the Spiral itself. As the research continued the patterns became more intricate; the answers seemed to lie in a mathematical mosaic.

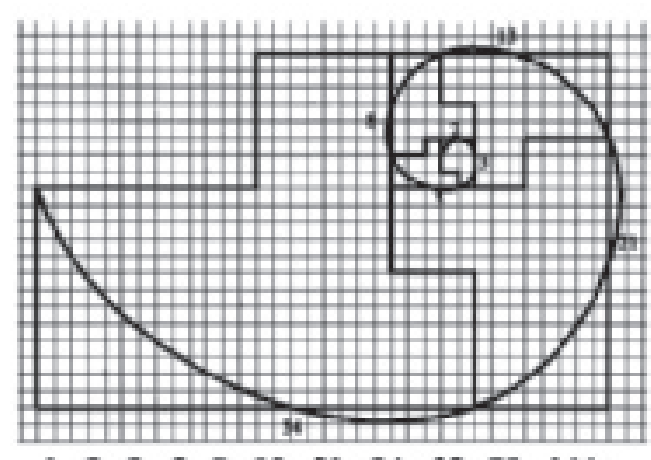

$1,2,3,5,8,13,21,34,55,89,144$

Fig 7: Fractal Patterning

We came across a fascinating idea from an American mathematician called Robert Ammann. He discovered a 'unit' of tiling, of three different interlocking but related shapes - the tiles had a special and subtle property, each one made up of the other two shapes along with a reduced version of itself. They fitted according to a set of exact rules. Though the pattern produced by these tiles looks similar, the pattern never repeats, it is aperiodic (Fig 6).

Dimensions for the Ammann tiles are derived from a hidden set of meshing grids that run diagonal to the tiles, beneath the surface of the pattern. The surprise is that these hidden lines form rectangles of length to breadth ratio equal to that of the golden section. The unique property the golden rectangle has of removing a square from it to leave behind a smaller rectangle that has the same proportions of the original rectangle, is relevant to the idea of self-similarity. It is also the root property of aperiodicity, a fundamental quality of the more complex behaviour of fractal patterns (Fig 7 ). 

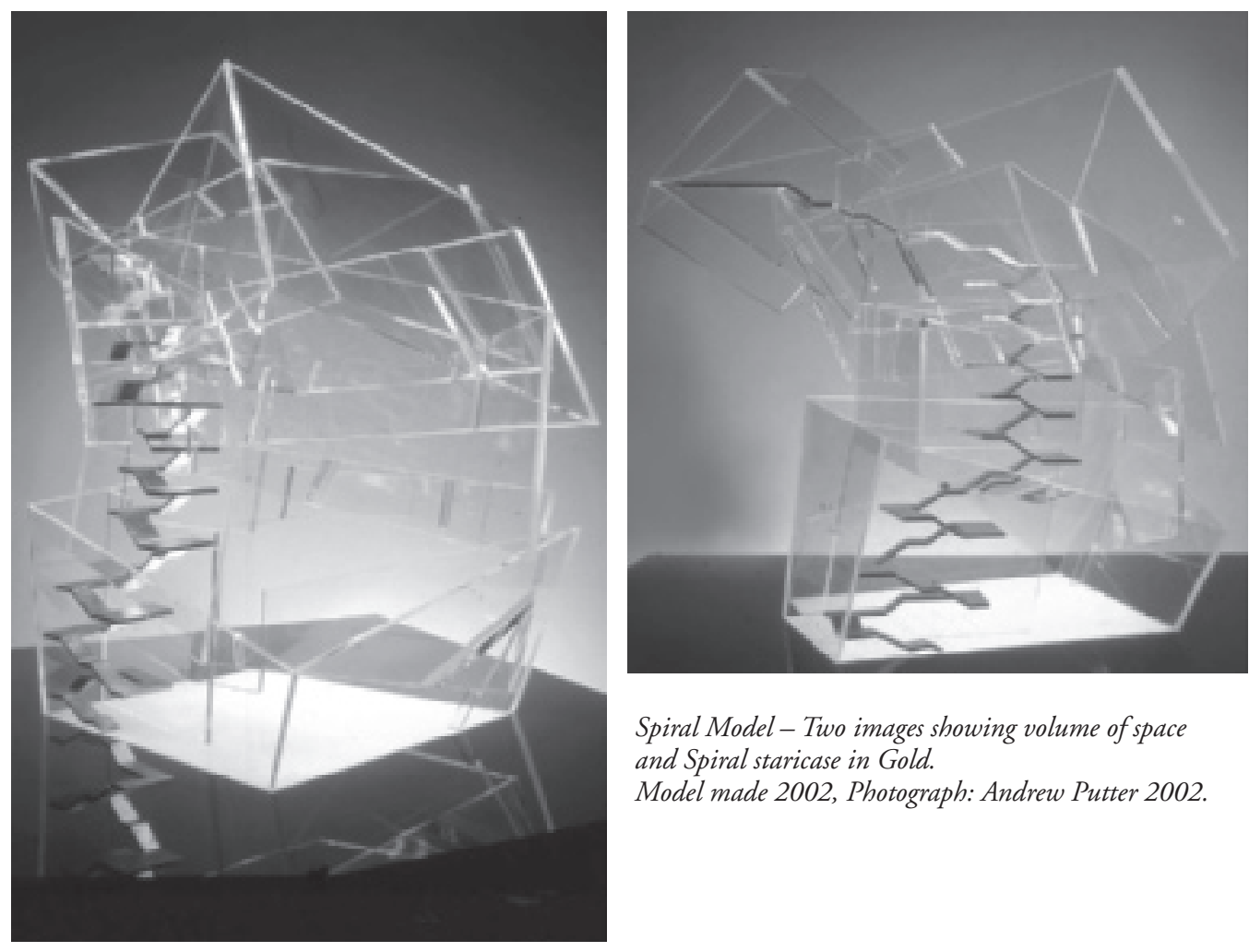

Spiral Model - Two images showing volume of space and Spiral staricase in Gold.

Model made 2002, Photograph: Andrew Putter 2002.

Since a fractal is scaleless, the folded-out strip of the V\&A spiral walls may be superimposed over the derived pattern. The strip can then be pulled away 'picking 'up part of the fractal it has contact with and be folded back on itself. The walls are now tiled. We used the word fractile to combine the mathematics of a fractal pattern and the concept of tiling.

\section{REFERENCE}

Balmond, Cecil (with Jannuzzi Smith) 'Informal' 2002, Prestel

Cecil Balmond

Director, Arup Group Board Ltd

Chairman, Arup Europe Division

Ove Arup \& Partners Ltd 